\title{
Can citizen science analysis of camera trap data be used to study reproduction? Lessons from Snapshot Serengeti program
}

\author{
Authors: Thel, Lucie, Chamaillé-Jammes, Simon, Keurinck, Léa, \\ Catala, Maxime, Packer, Craig, et al. \\ Source: Wildlife Biology, 2021(2) \\ Published By: Nordic Board for Wildlife Research
}

URL: https://doi.org/10.2981/wlb.00833

BioOne Complete (complete.BioOne.org) is a full-text database of 200 subscribed and open-access titles in the biological, ecological, and environmental sciences published by nonprofit societies, associations, museums, institutions, and presses.

Your use of this PDF, the BioOne Complete website, and all posted and associated content indicates your acceptance of BioOne's Terms of Use, available at www.bioone.org/terms-of-use.

Usage of BioOne Complete content is strictly limited to personal, educational, and non - commercial use. Commercial inquiries or rights and permissions requests should be directed to the individual publisher as copyright holder.

BioOne sees sustainable scholarly publishing as an inherently collaborative enterprise connecting authors, nonprofit publishers, academic institutions, research libraries, and research funders in the common goal of maximizing access to critical research. 


\title{
Can citizen science analysis of camera trap data be used to study reproduction? Lessons from Snapshot Serengeti program
}

\author{
Lucie Thel, Simon Chamaillé-Jammes, Léa Keurinck, Maxime Catala, Craig Packer, \\ Sarah E. Huebner and Christophe Bonenfant
}

L. Thel (https://orcid.org/0000-0002-2323-1106) ■ (lucie.mc.thel@gmail.com), L. Keurinck (https://orcid.org/0000-0001-6763-3601), M. Catala (https://orcid.org/0000-0003-1823-3665) and C. Bonenfant (https://orcid.org/0000-0002-9924-419X), Laboratoire de Biométrie et Biologie Evolutive, Unité Mixte de Recherche 5558, Bâtiment 711, Université Lyon I, Villeurbanne Cedex, France. - S. Chamaillé-Jammes (https://orcid.org/0000-0003-0505-6620), CEFE, Univ. Montpellier, CNRS, EPHE, IRD, Université Paul Valéry Montpellier 3, Montpellier, France, and: Mammal Research Inst., Dept of Zoology and Entomology, Univ. of Pretoria, Pretoria, South Africa. - SC-J and CB also at: LTSER France, Zone Atelier 'Hwange', Hwange National Park, Dete, Zimbabwe-CNRS HERD (Hwange Environmental Research Development) program. - C. Packer (https:/lorcid.org/0000-0002-3939-8162) and S. E. Huebner (https:/lorcid.org/0000-0001-5682-6467), College of Biological Sciences, Univ. of Minnesota, Saint Paul, MN, USA.

\begin{abstract}
Ecologists increasingly rely on camera-trap data to estimate biological parameters such as population abundance. Because of the huge amount of data camera trap can generate, the assistance of non-scientists is often sought after, but an assessment of the data quality is necessary. We tested whether volunteers data from one of the largest citizen science projects - Snapshot Serengeti - could be used to study breeding phenology. We tested whether the presence of juveniles (less than one or 12 months old) of species of large herbivores in the Serengeti: topi, kongoni, Grant's gazelle, could be reliably detected by the 'naive' volunteers versus trained observers. We expected a positive correlation between the proportion of volunteers identifying juveniles and their effective presence within photographs, assessed by the trained observers. The agreement between the trained observers was good (Fleiss' $\kappa>0.61$ for juveniles of less than one and 12 month(s) old), suggesting that morphological criteria can be used to determine age of juveniles. The relationship between the proportion of volunteers detecting juveniles less than a month old and their actual presence plateaued at 0.45 for Grant's gazelle, reached 0.70 for topi and 0.56 for kongoni. The same relationships were much stronger for juveniles younger than 12 months, reaching 1 for topi and kongoni. The absence of individuals $<$ one month and the presence of juveniles $<12$ months could be reliably assumed, respectively, when no volunteer and when all volunteers reported a presence of a young. In contrast, the presence of very young individuals and the absence of juveniles appeared more difficult to ascertain from volunteers' classification, given how the classification task was presented to them. Volunteers' classification allows a moderately accurate but quick sorting of photograph with/without juveniles. We discuss the limitations of using citizen science camera-traps data to study breeding phenology, and the options to improve the detection of juveniles.
\end{abstract}

Keywords: African ungulates, age determination, Alcelaphus cokii, Damaliscus jimela, Nanger granti

Camera trapping is increasingly used for ecological monitoring due to its low cost, relative ease of use and the variety of data it can supply (O'Connell et al. 2010). For instance, camera trap data are used to study species' occupancy and co-occurrence (Anderson et al. 2016), population dynamics (Karanth et al. 2006) or individual behaviour (e.g. vigilance behaviour: Chamaillé-Jammes et al. 2014, or diel activity patterns: Luo et al. 2019). A potential drawback of camera

This work is licensed under the terms of a Creative Commons Attribution 4.0 International License (CC-BY) <http:// creativecommons.org/licenses/by/4.0/>. The license permits use, distribution and reproduction in any medium, provided the original work is properly cited. traps is the huge amounts of data that are generated $(>7$ millions photographs for the Snapshot Serengeti initiative alone). Ecologists have realized that the benefits of continuously collecting data in the field can quickly be negated by the burden of database management, and visual inspection and analysis of photographs to record the desired data (Wearn and Glover-Kapfer 2017).

To process such a massive amount of information in a reasonable time, scientists have sought the help of non-specialists who perform diverse tasks like counting objects in photographs, describing picture content or identifying animal and plant species (McShea et al. 2015). Initially, part of the scientific community was sceptical about citizen science, in particular questioning data quality (Riesch and Potter 
2014). However, volunteers have sometimes proven to be as efficient as experts, for instance for the identification of large herbivore species in savanna ecosystems (Swanson et al. 2016). More recently, the advances in deep learning have led computers to become as efficient as people at identifying species, and, sometimes, behaviour classification problems (Norouzzadeh et al. 2018). However, human judgment is still valuable in particular cases where too little data are available to train models (e.g. active learning, Joshi et al. 2009), or when differences among the objects to be classified are subtle and classification requires some subjectivity (Miele et al. 2021). We believe this is the case for age classification problems, for which to the best of our knowledge the number of precisely labelled pictures taken in the wild is currently too small to allow an efficient and reliable automatization of the process.

Under the assumption that the detectability of juveniles and adult females segments of the population is not biased by camera traps methodology, classifying individuals into ageclasses such as juveniles and adults would allow estimates of key demographic parameters (e.g. reproductive rates) or lifehistory traits (e.g. breeding phenology). For instance, Ogutu and colleagues (2008) highlighted that rainfall influences the abundance of several large herbivore species of the MaraSerengeti ecosystem, by acting differently on each segment of the populations at specific periods of the year. Furthermore, it would facilitate the study of the relationships between population characteristics and their environments such as between birth phenology, diet and food resource availability (Sinclair et al. 2000), or their potential evolution in the context of climate change (Visser and Both 2005). Until now, the study of those key demographic parameters has been mainly conducted by direct field observations (Côté and Festa-Bianchet 2001 in mountain goats, Plard et al. 2013 in roe deer). However, this methodology still requires an intensive and often costly field effort. Identifying and counting juveniles from camera traps could reduce this field effort, or allow larger-scale or longer-term studies, as suggested by Hofmeester et al. (2020), but could also be time-consuming because of tedious data processing. With the help of citizen science, data handling time could be substantially reduced, but the accuracy of non-specialists in detecting juveniles of large mammals from photographs has not yet been explored.

Here, we evaluate the usefulness of camera trap data annotated by citizen scientists online to assess the presence of juveniles of large herbivores in the photographs. We use photographs and citizen classifications from the Snapshot Serengeti project (Swanson et al. 2015), one of the world's largest citizen science programs, on a subset of the data. We focus on the detection of juveniles in three species found in the Serengeti National Park, Tanzania with contrasting social and neonatal behaviours: topi Damaliscus jimela, kongoni Alcelaphus cokii and Grant's gazelle Nanger granti. We first evaluate the agreement between trained observers from our research team, and then test the ability of the volunteers to detect juveniles by comparing their classification with ours. We predict a better agreement between trained observers for the youngest age class because determination criteria are clearer and easier to identify than for older juveniles (e.g. absence of horns). Consequently, the level of agreement should decrease for age classes that are based on more subjective or difficult-to-assess criteria (e.g. shape of the horns). Under the hypothesis that volunteers could generally identify juveniles correctly, we expect a positive relationship between the proportion of volunteers reporting a juvenile on a photograph and the probability of the actual presence of a juvenile, as determined by the trained observers. Again, we expect the correlation to be stronger for the youngest age class of juveniles because they are easier to differentiate from adults. Across species, we expect a higher agreement and correlation for topi and kongoni than for Grant's gazelle because the former are larger, live in smaller groups and have similar body growth rate between males and females (Wilson and Mittermeier 2011), hence reducing the risk of confusion between young males and older females. Overall, our study details the strengths and weaknesses of camera trap data, in particular when classified by citizen scientists, for the study of reproductive traits such as reproductive rates or breeding phenology.

\section{Material and methods}

\section{Study site}

The surveyed area within the Serengeti National Park, Tanzania, is composed of open plains and savanna woodlands. Rain mostly occurs between November and June (wet season), with mean annual rainfall increasing from $500 \mathrm{~mm}$ in the southeast to $1100 \mathrm{~mm}$ in the northwest. This area harbours a rich community of large herbivores, composed of gregarious and migratory wildebeest Connochaetes mearnsi, zebra Equus sp. and Thomson's gazelle Eudorcas nasalis, but also resident populations such as Cape buffalo Syncerus caffer or warthog Phacochoerus africanus (Sinclair and Norton-Griffiths 1995). Community dynamics are driven both by herbivores, maintaining an open state of the grassland by intensive grazing (McNaughton 1985, Sinclair and Norton-Griffiths 1995) as well as large predators (e.g. lion Panthera leo and hyena Crocuta crocuta, Sinclair and Norton-Griffiths 1995).

\section{Camera trap data}

The Snapshot Serengeti camera trap grid was deployed in 2010 in Serengeti National Park, Tanzania, to monitor lions and their prey, though the bycatch of numerous other species has proven useful as well. Running continuously since 2010 , the grid spans $1125 \mathrm{~km}^{2}$ in the center of the park. We used data provided by Snapshot Serengeti camera survey recorded between July 2010 and April 2013 (Supporting information 1). The camera traps were set $-50 \mathrm{~cm}$ above ground in the centre of a $5 \mathrm{~km}^{2}$ grid cell. The detection radius was approximately $45^{\circ}$ and their field of view about $14 \mathrm{~m}$ (Swanson et al. 2015). Cameras took a rapid series of three pictures upon trigger of the motion and heat sensors ('capture event' in Swanson et al. 2015, hereafter called a 'sequence' following Meek et al. 2014) in a few seconds interval, with a oneminute delay between sequences. 


\section{Choice of studied species and sorting steps of the dataset}

Among the many large herbivore species present in the study site, we selected topi, kongoni and Grant's gazelle due to their contrasting biology and characteristics useful to assess the age classes of individuals. The criteria considered were 1) number of available sequences, 2) relatively small group size, 3) presence of horns in males and females, 4) relatively large size of the young (young of larger species are larger, and therefore criteria like horns are easier to detect), 5) contrasting anti-predator of behaviour of the young (Supporting information 2).

We selected the final dataset ( $\mathrm{n}=2359$ sequences) to conduct the analyses following several sorting steps, based on the detection of the species of interest and of the presence of juveniles from the initial complete dataset $(n=1184657$ sequences) by the volunteers. We then corrected this dataset thanks to the trained observers reclassifications (details in Table 1).

\section{Assessing the presence or absence of juveniles in photographs}

All Snapshot Serengeti photographs have been uploaded to the online citizen science platform 'The Zooniverse' (<www.zooniverse.org $>$ ) to be classified by volunteers. Each sequence was processed by as many as 25 volunteers $($ minimum $=11$, maximum $=57$, Swanson et al. 2016), who each identified what species was present in the sequence and recorded whether at least one juvenile was present or not. Note that no formal definition of a juvenile was provided to volunteers, nor were there any particular guidelines about how to identify a juvenile. Volunteers simply searched and checked 'young' in the Zooniverse interface when they suspected the presence of non-adult individuals. Regarding age classes, the only question volunteers had to reply to was: 'Are there any young present?'. For each sequence, the volunteers' classifications were then compiled via a plurality algorithm to yield a consensus classification, leading to a proportion of volunteers $(P v)$ who identified at least one juvenile in each sequence (details in Swanson et al. 2015). Here, we used all sequences where volunteers identified topi, kongoni and Grant's gazelle, with at least one volunteer $(P v>0 \%)$ having annotated the presence of a juvenile. We could not analyse all sequences for which no volunteer had reported a juvenile, as these were too many $(n=2018,11141$ and 6628 for topi, kongoni and Grant's gazelle respectively) to be reviewed individually. However, we checked a subset of them ( $n=1000$ for each species), and the chance that a trained observer observed a 'true' juvenile (i.e. of less than 12 months old, see age classes definition below) was $<6.5 \%$ for all three species studied when no volunteer did report one. We did not correct observations for recaptures of the same individuals as we were only interested in the ability of volunteers to detect the presence of juveniles on the sequences, but not the actual number of juveniles.

Three of us (LT, LK and MC), considered here as trained observers, searched all sequences retained for juveniles, which were assigned to an age class when detected. We used previously published morphological descriptions of the studied species (e.g. shape and size of horns, size relating to the adult; Supporting information 3) to identify and age individuals. We distinguished between 1) juveniles < one month, 2) between one and six months, 3) between six and 12 months, 4) between 12 and 24 months, termed yearling hereafter and 5) individuals over two years old, termed adults hereafter. We defined age classes according to biological characteristics relevant to juvenile identification for each species (e.g. very young individuals for birth phenology identification, juveniles under one year for recruitment estimation). We recorded observers' classifications with Aardwolf software (Krishnappa and Turner 2014). Ultimately, we produced a dataset describing the presence or absence $\left(M_{i, s, j}\right)$, in each sequence of individuals of any of the five age categories $i$, for the species $s$, by trained observer $j$.

Table 1. Number of sequences at each sorting step from the extraction of raw data to the selection of all the independent sequences with at least one individual $<1$ month old, for the three species of the study: topi, kongoni and Grant's gazelle (pictures from Snapshot Serengeti program, Tanzania, between July 2010 and April 2013). Standard deviations are calculated on the basis of the classifications of the three trained observers.

\begin{tabular}{|c|c|c|c|c|c|c|}
\hline \multirow[b]{2}{*}{ Step no. } & \multirow[b]{2}{*}{ Step name } & \multirow[b]{2}{*}{ Consists in } & \multirow[b]{2}{*}{ Classifier } & \multicolumn{3}{|c|}{ Number of sequences } \\
\hline & & & & Topi & Kongoni & $\begin{array}{l}\text { Grant's } \\
\text { gazelle }\end{array}$ \\
\hline 1 & Row data & all sequences produced during the survey & none & 1184657 & 1184657 & 1184657 \\
\hline 2 & Blank sorting & sequences with animals & volunteers & 319915 & 319915 & 319915 \\
\hline 3 & Species sorting 1 & sequences with study species & volunteers & 2299 & 12431 & 7723 \\
\hline 4 & Young sorting 1 & $\begin{array}{l}\text { sequences with at least more than } 0 \% \text { of } \\
\text { volunteers identifying young }\end{array}$ & volunteers & 281 & 1290 & 1095 \\
\hline 5 & Species sorting 2 & $\begin{array}{l}\text { sequences with study species corrected by } \\
\text { trained observers }\end{array}$ & trained observers & 324 & 1281 & 754 \\
\hline 6 & Young sorting 2 & sequences with at least one young & trained observers & $216 \pm 1$ & $830 \pm 17$ & $348 \pm 18$ \\
\hline 7 & Young sorting 3 & $\begin{array}{l}\text { sequences with at least one young less than } \\
\text { one month old }\end{array}$ & trained observers & $59 \pm 9$ & $137 \pm 33$ & $58 \pm 3$ \\
\hline 8 & Re-sight sorting 1 & $\begin{array}{l}\text { independent sequences (remove of resights } \\
\text { notified by the observers) }\end{array}$ & trained observers & $39 \pm 7$ & $71 \pm 27$ & $50 \pm 5$ \\
\hline $8^{\prime}$ & Re-sight sorting 2 & $\begin{array}{l}\text { independent sequences (remove of sequences } \\
\text { taken less than } 10 \text { minutes after the } \\
\text { previous one by the same camera trap) }\end{array}$ & theoretical sorting & $42 \pm 8$ & $70 \pm 22$ & $40 \pm 4$ \\
\hline
\end{tabular}




\section{Statistical analyses}

We first evaluated the agreement between the three trained observers on the detection of individuals assigned to each age class for each species. We measured this agreement with the Fleiss' $\kappa$, implemented in the 'raters' $\mathrm{R}$ package (Quatto and Ripamonti 2014). Fleiss' $\kappa$ (Fleiss 1971) is the comparison of agreement between 2+ judges and the level of agreement expected by chance alone. It takes values between -1 and 1 , values $<0$ indicating an agreement lower to what could be expected by chance, values $>0$ indicating a greater agreement than expected by chance, and values $=0$ indicating an agreement close to random. We tested for significance of the difference between the Fleiss' $\kappa$ using a bootstrap procedure following Vanbelle and Albert (2008) (Supporting information 4).

We tested the relationship between the proportion of volunteers identifying at least one juvenile $(P v)$ and the probability that trained observers had identified at least one juvenile $<1$ month (category $i=1$ ). We also explored the same relationship with juveniles $<12$ months (therefore including juveniles of categories 1-3 above). We fitted three generalized estimating equation models: one linear (Eq. 1 below) and two piecewise models. The first piecewise model was characterized by a slope on both sides of the threshold (Eq. 2), the second by a slope before and a plateau after the threshold (Eq. 3). We fitted piecewise models to search for a potential 'saturation' phenomenon, whereby beyond a specific proportion of volunteers the probability to effectively observe a juvenile does not increase anymore. We also fitted the null model for comparison. All the models were fitted for the two age classes and for each species individually, using the wgee function implemented in the 'wgeesel $\mathrm{R}$ package (Xu et al. 2018). We selected the best model using the Quasi-likelihood under the independence model criterion QIC (thresholds selected by comparison of QIC of the models for each species and age class as well). It is a modification of the Akaike information criterion AICc, suitable when quasi-likelihood is used instead of likelihood (Pan 2001), implemented in the 'MuMIn' R package (Bartoń 2019). We used a logit link function and a binomial distribution of errors (Agresti 2002), considering the proportion of volunteers identifying at least one juvenile as fixed effect, and the identity of the sequence as clustering variable with an exchangeable correlation structure:

$$
\operatorname{logit}\left(M_{s, i, j}\right)=\mu+\beta_{s, i} \times P v_{s} \quad \text { (Eq. } 1-\text { linear model) }
$$

and

$$
\operatorname{logit}\left(M_{s, i, j}\right)=\left\{\begin{array}{l}
\mu+\beta 1_{s, i} \times P v_{s} \text { if } P v_{s} \leq \theta \\
\mu+\beta 2_{s, i} \times P v_{s} \text { if } P v_{s}>\theta
\end{array}\right. \text { (Eq. 2-piecewise model 1) }
$$

and

$$
\operatorname{logit}\left(M_{s, i, j}\right)=\left\{\begin{array}{c}
\mu+\beta 1_{s, i} \times P v_{s} \text { if } P v_{s} \leq \theta \\
\mu 2 \text { if } P v_{s}>\theta
\end{array} \quad(\text { Eq. } 3-\text { piecewise model 2) }\right.
$$

where $M_{s, i j,}$ denotes the presence or absence of at least one juvenile of the age class $i$ ( $<1$ or 12 months) for a given sequence, for the species $s$ and the observer $j, \mu$ is the expected mean probability of actual presence of at least one juvenile of the age class $i$ when none of the volunteers identified the presence of a juvenile, $P v_{s}$ is the proportion of volunteers identifying at least one juvenile for a given sequence, for the species $s$ and $\theta$ is the best threshold. We performed all analyses using the R statistical software (<www.r-project.org $>$ ).

\section{Results}

From the Snapshot Serengeti monitoring program, we obtained 281, 1290 and 1095 sequences for topi, kongoni and Grant's gazelle respectively (Fig. 1a) sorting step 'Young sorting 1' and Table 1. Our selection process led to a dramatic drop in the number of usable sequences (i.e. those containing at least one $<1$ month old juvenile), with a tenfold reduction at the species level: it only remained between $7.7 \%$ and $18.1 \%$ of the complete dataset available for the species $(n=59 \pm 9$ SD, $137 \pm 33$ SD and $58 \pm 3$ SD for each species respectively, Fig. 1a) sorting step young sorting 3 ' and Table 1. The largest losses of sequences happened during the phases of selection of sequences containing the studied species and then juveniles (Fig. 1a and Table 1). Figure $1 \mathrm{~b}$ suggests that we would obtain similar results concerning the loss of sequences after selection for sequences containing juveniles for any large herbivore species recorded in the Snapshot Serengeti program.

The three trained observers identified $993 \pm 49$ SD, 3020 \pm 180 SD and $2128 \pm 224$ SD individuals of any age class. On average, they could not assign an age class to $-11 \%$ $(n=118 \pm 68$ SD $),-20 \%(n=775 \pm 256$ SD $)$ and $32 \%$ $(n=1026 \pm 492$ SD) of the individuals for topi, kongoni and Grant's gazelle respectively, a significant between-species difference $\left(\chi^{2}=262.83, \mathrm{df}=2, \mathrm{p}<0.001\right)$.

For all species, and as expected, the agreement between trained observers was highest for the youngest age class, then declined with age until the yearling class was reached (Fig. 2). Yearlings were reasonably well classified in kongoni, but overly misclassified in topi and Grant's gazelle (Fig. 2). As expected, the agreement among trained observers was the highest for the youngest age class of juveniles, but also for the second age class and the adults, with Fleiss' $\kappa$ values almost always $>0.61$ (denoting a substantial agreement, following Landis and Koch 1977), except for Grant's gazelle aged between one and six months and adult topi. Agreement among observers was the highest for topi aged $<1$ month old (Fleiss' $\kappa=0.78$ [0.72;0.84]). In support of our prediction, we observed the lowest agreement for juveniles aged six months and older, and more obviously so for Grant's gazelle. Agreement among observers concerning the three first age classes pooled together (representing the juveniles) was very good, with Fleiss' $\kappa$ values largely $>0.61$ for all the species. The same holds true for juveniles between one and 12 months old when pooled together. Our results were globally consistent among the three species studied, with the highest agreement for topi, and the least for Grant's gazelle (Fig. 2). All estimated Fleiss' $\kappa$ were significantly different from an 

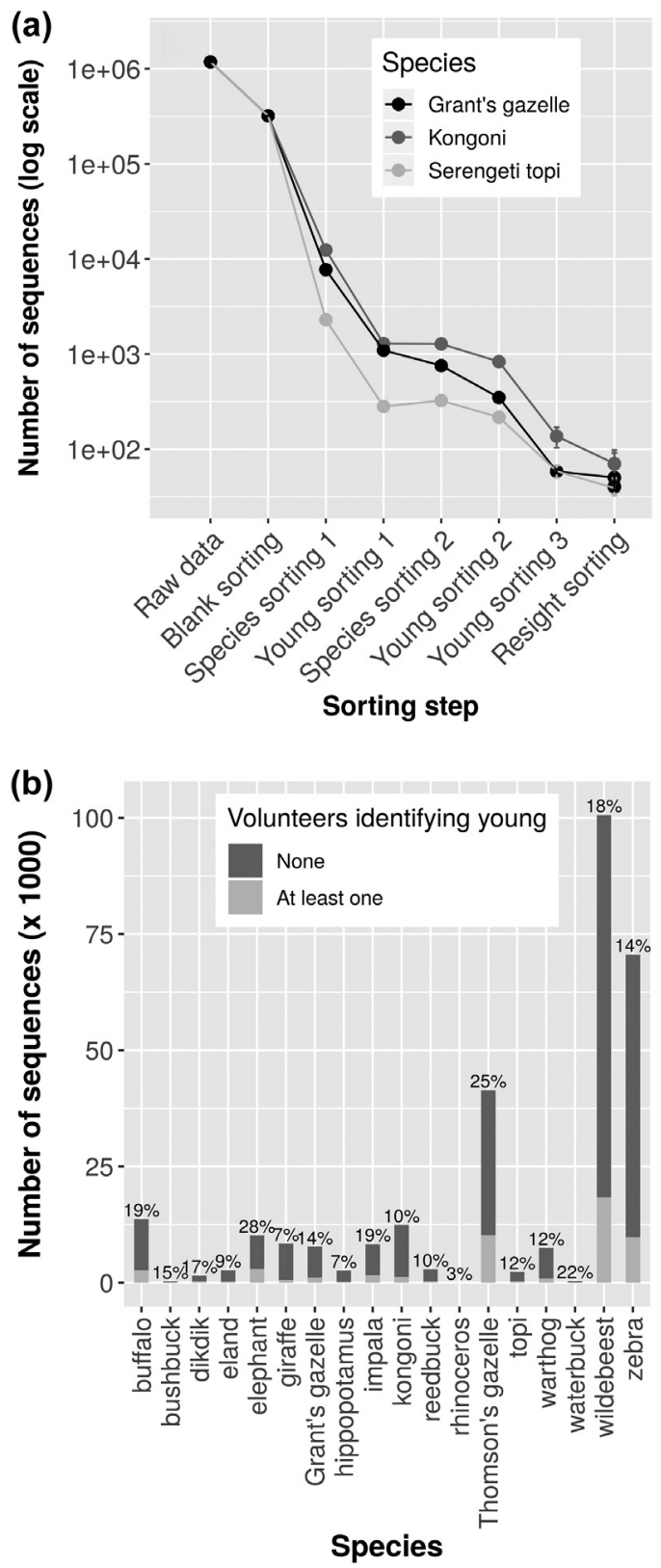

Figure 1. (a) Number of sequences available at each sorting step from the extraction of raw data to the selection of all the independent sequences with at least one individual $<1$ month old, for the three species of the study: topi, kongoni, Grant's gazelle. Pictures from Snapshot Serengeti, Tanzania, between July 2010 and April 2013. Raw data: all sequences produced during the survey; blank sorting: sequences with animals; species sorting 1: sequences with study species according to the volunteers; young sorting 1 : sequences with at least one volunteer identifying 'young'; species sorting 2: sequences with study species corrected by trained observers; young sorting 2: sequences with at least one young according to the trained observers; young sorting 3: sequences with at least one young $<1$ month old according to the trained observers; resight sorting: independent sequences once sequences taken less than 10 minutes after the previous one by the same camera trap and presenting the same species have been removed (following Palmer et al. 2018). Note the log scale for the ordinate axis, vertical bars represent the standard deviations. (b) Number of sequences available for all the large herbivore species present in the study site. The proportions indicate the proportion of sequences where at least one volunteer identified juveniles for a given species.

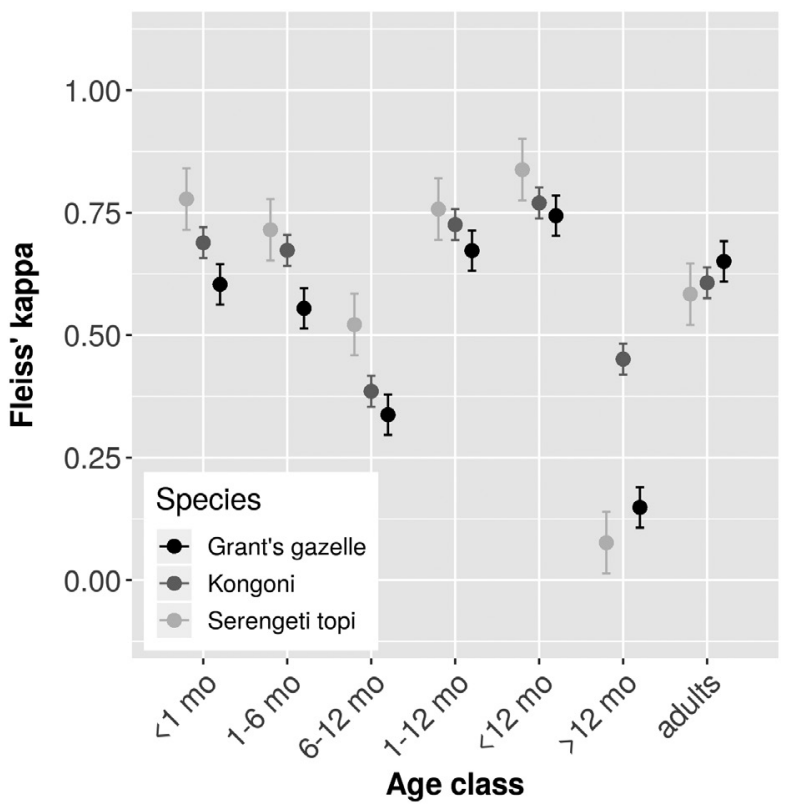

Figure 2. Fleiss' $\kappa$ denoting the level of agreement between the three trained observers (LT, LK and MC) in the identification of presence or absence of at least one individual belonging to any of the five age classes ( $<1$ month, 1-6 months, 6-12 months, $>12$ months, adults), for the three species of interest. Pictures from Snapshot Serengeti Program, Tanzania, between July 2010 and April 2013. The two combined age classes ' $1-12$ months' and ' $<12$ months' are also presented. Light grey dots: topi, dark grey dots: kongoni, black dots: Grant's gazelle. Vertical bars represent the confidence intervals of the points estimates. A Fleiss' $\kappa$ near 1 denotes an almost perfect agreement, whereas a value near or $<0$ means a very poor agreement between raters.

agreement obtained by chance ( $\mathrm{p}$-values $<0.001$, see Supporting information 4).

The model best describing our data for the age class $<1$ month was the linear model for topi, the piecewise model with slopes on both sides of the threshold for kongoni and the piecewise model with slope before and plateau after the threshold for Grant's gazelle (Table 2). The model best describing our data for the age class $<12$ months was the threshold model with slopes on both sides of the threshold for topi, kongoni and Grant's gazelle (Table 2).

The probability of observing a juvenile when all volunteers reported one was near 1 for juveniles $<12$ months for topi and kongoni. Concerning Grant's gazelle, this probability only reached 0.90 when $41 \%$ of the volunteers recorded the presence of young. Between $41 \%$ and $100 \%$ of volunteers identifying young in the sequences, the probability decreased (Fig. 3d-f). When investigating the presence of juveniles $<1$ month, the probability that a juvenile was actually present was never greater than $70 \%$ (Fig. 3a-c). On the other hand, the model predicted that when no volunteer reported the presence of juveniles, the probability that there was juveniles $<1$ month was under $1.8 \%$, but there was at least $9.6 \%$ chance to observe a juvenile $<12$ months.

Contrary to our expectations, the piecewise model characterized by two slopes was almost always selected as the best model for the three species and both age classes. This denotes a sudden change in the rate of detection from a cer- 
Table 2. Statistics of models investigating the relationship between the proportion of volunteers identifying at least one 'young' and the probability of presence of at least one individual $<1$ or 12 months, assessed by the trained observers on a given sequence for the three species of interest: topi, kongoni and Grant's gazelle. Best models are in bold. $\theta=$ estimated threshold (\% of volunteers), QIC=Quasi-likelihood under the Independence model Criterion, estimates \pm standard error.Best models are in bold type.

\begin{tabular}{|c|c|c|c|c|c|c|}
\hline Age class & Species & Model type & $\theta$ & QIC & Quasi-likelihood & Estimates \\
\hline \multirow[t]{12}{*}{$<1$ month } & \multirow[t]{4}{*}{ Topi } & Null & & 924.6 & -459.77 & $\mu=-1.509 \pm 0.133$ \\
\hline & & Linear & & 630.9 & -311.05 & $\begin{array}{r}\mu=-4.013 \pm 0.342 \\
\beta=0.048 \pm 0.005\end{array}$ \\
\hline & & Piecewise - slope/plateau & 90 & 635.2 & -313.23 & $\begin{array}{c}\mu=-4.102 \pm 0.362 \\
\beta 1=0.051 \pm 0.005 \\
\mu 2=0.626[0.523 ; 0.720]\end{array}$ \\
\hline & & Piecewise - slope/slope & 78 & 634.7 & -310.49 & $\begin{array}{l}\mu=-8.863 \pm 1.763 \\
\beta 1=0.045 \pm 0.007 \\
\beta 2=0.064 \pm 0.023\end{array}$ \\
\hline & \multirow[t]{4}{*}{ Kongoni } & Null & & 2614.4 & -1304.83 & $\mu=-2.125 \pm 0.081$ \\
\hline & & Linear & & 1993.0 & -992.05 & $\begin{array}{r}\mu=-3.977 \pm 0.174 \\
\beta=0.043 \pm 0.003\end{array}$ \\
\hline & & Piecewise - slope/plateau & 90 & 2005.8 & -998.42 & $\begin{array}{c}\mu=-4.007 \pm 0.178 \\
\beta 1=0.044 \pm 0.003 \\
\mu 2=0.498[0.434 ; 0.563]\end{array}$ \\
\hline & & Piecewise - slope/slope & 10 & 1966.7 & -977.58 & $\begin{array}{r}\mu=-10.522 \pm 1.740 \\
\beta 1=0.687 \pm 0.178 \\
\beta 2=0.039 \pm 0.003\end{array}$ \\
\hline & \multirow[t]{4}{*}{ Grant's gazelle } & Null & & 1236.2 & -615.91 & $\mu=-2.479 \pm 0.117$ \\
\hline & & Linear & & 1016.6 & -501.81 & $\begin{array}{r}\mu=-3.653 \pm 0.181 \\
\beta=0.055 \pm 0.007\end{array}$ \\
\hline & & Piecewise - slope/plateau & 43 & 969.7 & -480.75 & $\begin{aligned} \mu= & -4.355 \pm 0.227 \\
\beta 1 & =0.097 \pm 0.008 \\
\mu 2= & 0.451[0.353 ; 0.552]\end{aligned}$ \\
\hline & & Piecewise - slope/slope & 43 & 974.6 & -480.48 & $\begin{array}{l}\mu=-4.573 \pm 0.544 \\
\beta 1=0.094 \pm 0.010 \\
\beta 2=0.006 \pm 0.013\end{array}$ \\
\hline \multirow[t]{12}{*}{$<12$ months } & \multirow[t]{4}{*}{ Topi } & Null & & 1241.3 & -618.00 & $\mu=0.698 \pm 0.111$ \\
\hline & & Linear & & 948.0 & -468.64 & $\begin{array}{r}\mu=-0.866 \pm 0.184 \\
\beta=0.059 \pm 0.007\end{array}$ \\
\hline & & Piecewise - slope/plateau & 60 & 964.6 & -477.23 & $\begin{array}{c}\mu=-0.987 \pm 0.191 \\
\beta 1=0.069 \pm 0.007 \\
\mu 2=0.958[0.926 ; 0.977]\end{array}$ \\
\hline & & Piecewise - slope/slope & 30 & 946.5 & -464.45 & $\begin{array}{l}\mu=-3.202 \pm 0.624 \\
\beta 1=0.031 \pm 0.017 \\
\beta 2=0.090 \pm 0.022\end{array}$ \\
\hline & \multirow[t]{4}{*}{ Kongoni } & Null & & 4992.3 & -2493.63 & $\mu=0.609 \pm 0.054$ \\
\hline & & Linear & & 3726.5 & -1858.95 & $\begin{array}{r}\mu=-1.139 \pm 0.108 \\
\beta=0.089 \pm 0.006\end{array}$ \\
\hline & & Piecewise - slope/plateau & 90 & 3726.6 & -1859.03 & $\begin{array}{r}\mu=-1.140 \pm 0.108 \\
\beta 1=0.089 \pm 0.006 \\
\mu 2=0.999[0.997 ; 1]\end{array}$ \\
\hline & & Piecewise - slope/slope & 12 & 3684.8 & -1835.45 & $\begin{array}{l}\mu=-3.041 \pm 0.271 \\
\beta 1=0.213 \pm 0.029 \\
\beta 2=0.066 \pm 0.006\end{array}$ \\
\hline & \multirow[t]{4}{*}{ Grant's gazelle } & Null & & 3127.4 & -1561.20 & $\mu=-0.154 \pm 0.067$ \\
\hline & & Linear & & 2940.5 & -1463.22 & $\begin{array}{r}\mu=-0.837 \pm 0.140 \\
\beta=0.049 \pm 0.010\end{array}$ \\
\hline & & Piecewise - slope/plateau & 30 & 2866.2 & -1428.27 & $\begin{array}{c}\mu=-1.285 \pm 0.132 \\
\beta 1=0.091 \pm 0.009 \\
\mu 2=0.809[0.748 ; 0.859]\end{array}$ \\
\hline & & Piecewise - slope/slope & 41 & 2866.4 & -1425.59 & $\begin{array}{r}\mu=-0.009 \pm 0.506 \\
\beta 1=0.083 \pm 0.010 \\
\beta 2=-0.029 \pm 0.013\end{array}$ \\
\hline
\end{tabular}

tain percentage of volunteers. The detection rate decreases for kongoni and Grant's gazelle for young $<12$ months beyond 12 and $41 \%$ of volunteers voting for the presence of young respectively. The number of volunteers who did classify a photograph was independent from the probability to detect a young.

\section{Discussion}

Our study reveals the strength and weaknesses of using citizen-based assessment of age classes on camera trap pictures. Clearly, citizen involvement through an online platform has been critical for the classification of the millions of photo- 

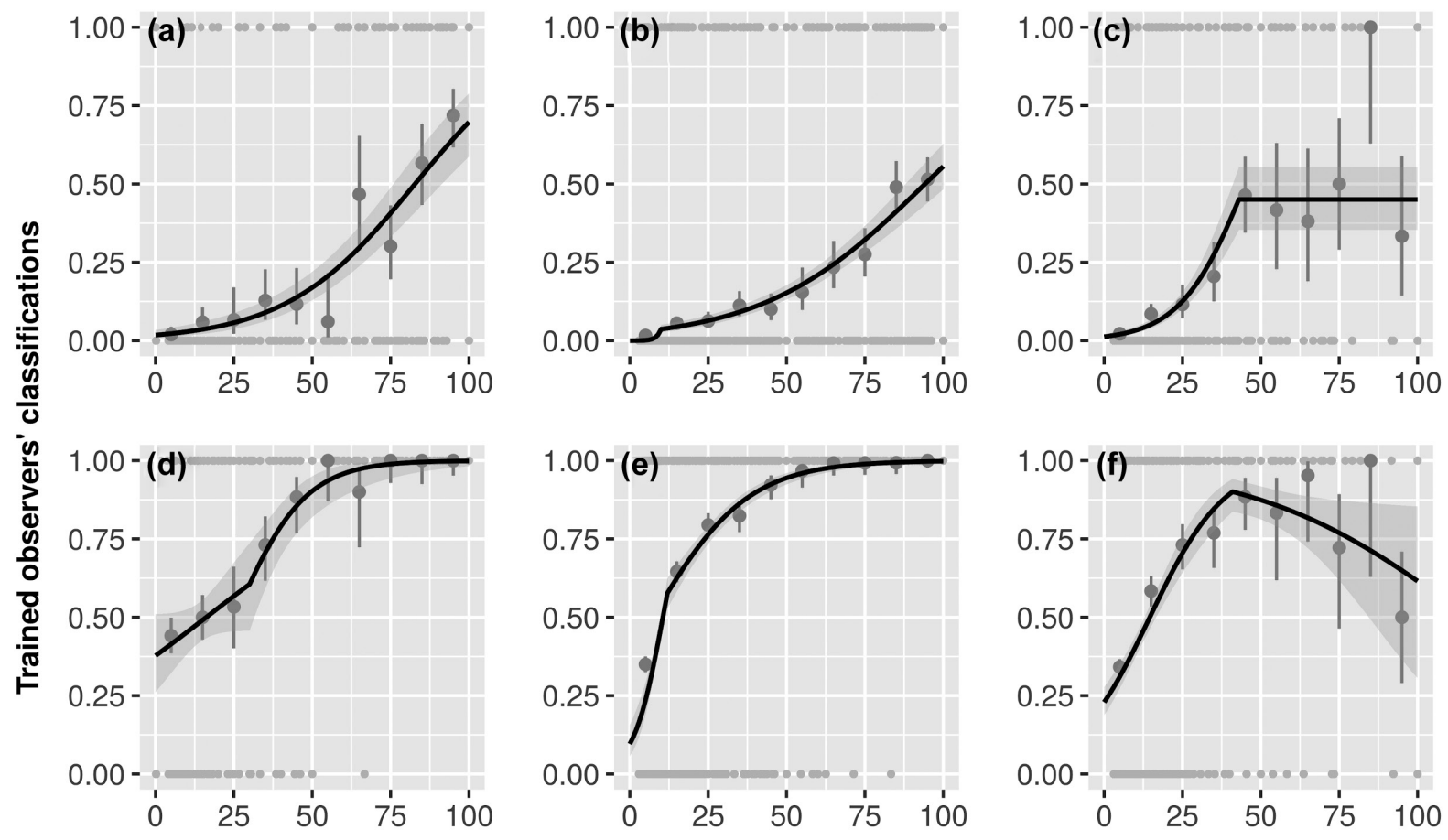

Proportion of volunteers

Figure 3. Relationship, as predicted from the best model (see text for details), between the proportion of volunteers identifying the presence of 'young' and the probability of presence of at least one individual $<1(\mathrm{a}-\mathrm{c})$ and $<12(\mathrm{~d}-\mathrm{f})$ months assessed by the trained observers in a given sequence of photographs ( $0<$ proportion of volunteers identifying 'young' $\leq 1)$, for the three species of interest: (a) and (d) topi, (b) and (e) kongoni and (c) and (f) Grant's gazelle. Pictures from Snapshot Serengeti Program, Tanzania, between July 2010 and April 2013. Light grey dots represent the probability of presence of at least one individual < selected age class in each sequence assessed by the three trained observers, dark grey dots represent the mean of those probabilities for each $10 \%$ volunteers interval, vertical bars represent $95 \%$ confidence intervals. Solid line represents predicted values from the best model. Shaded areas represent $95 \%$ confidence interval of these predicted values .

graphs collected by the Snapshot Serengeti initiative. Also, despite leaving it to the volunteers to decide what a juvenile looks like, volunteers' classification allows a rough, moderately accurate, but quick sorting of sequences with/without juveniles.

Our study makes clear that, for the species studied and given the minimal guidelines given to volunteers, the 'absence' of very young ( $<1$ month) individuals on pictures can be reliably assumed when no volunteer reports a presence. The sequences almost never labelled by volunteers as containing 'young' were very unlikely to contain young under one month of age according to the trained observers. This suggests that volunteers rarely miss very young juveniles. This likely occurs because very young juveniles are distinctively smaller than adults, and possibly also because very young mammals share some physical characteristics such as relatively large eyes, long legs, short and rounded nose, all belonging to 'Kindchenschema', known to be very attractive stimuli for humans (Brosch et al. 2007, Golle et al. 2013). By contrast, the 'presence' of very young individuals appears more difficult to ascertain from volunteers' data, and this apparently comes from the lack of guidelines given to volunteers. Indeed, consistent with the idea that volunteers easily identify very young individuals, a large consensus among volunteers around the presence of a very young juvenile could be a reliable indication of actual presence, but only when no older juveniles are present (compare Fig. 3a-c and Support- ing information 5 - Fig. A). Unfortunately, the absence or presence of older juveniles cannot currently be known without a reassessment of the pictures because volunteers were not asked to differentiate between juvenile age classes. Therefore, the presence of very young juveniles remains difficult to ascertain. On the other hand, the 'presence' of juveniles, irrespectively of their age, can be reliably assumed when all volunteers agree about a presence (Fig. 3a-c), especially for topi and kongoni: the model predicted that when all the volunteers reported the presence of juveniles, the probability that there was juveniles $<12$ months was indeed at least $99.8 \%$. This time, the 'absence' of juveniles regardless of their age is less accurate, meaning that they are missed quite often. As they grow, juveniles become increasingly similar to adults, and are more likely to be mistaken for the latter by volunteers. Anyhow, we emphasize that detectability of juveniles is not equal between species (Fig. 3).

Among the three trained observers, we found the best agreement in the detection of age classes for a given sequence for topi and kongoni, suggesting that they are the easiest species to classify, and Grant's gazelle the hardest. The combination of small body size and gregariousness could explain why the determination of age was more difficult and heterogeneous for Grant's gazelle than for the two other species. Topi and kongoni are fairly large, and the small body size of Grant's gazelle makes the detection of some inconspicuous age criteria challenging (e.g. presence or absence of very 
small horn buds on the forehead of the individuals). On the contrary, it is certainly easier to discriminate between adults and juveniles in the largest herbivores, such as giraffe Giraffa camelopardalis or elephant Loxodonta africana because of the marked size difference between them. Discrimination between several juvenile age classes will certainly be difficult still as individuals are often only partially spotted by camera traps and subtle aging criteria are not visible. More generally, in species forming large herds such as wildebeest, young might be particularly difficult to spot and are likely missed frequently. In large groups, the body of many individuals overlaps, hampering our ability to see aging criteria correctly, and to age them accurately. This could explain why in our study for instance, Grant's gazelle frequently occurring in large herds reaching more than 30 individuals in some sequences, is also the one with the lowest identification success of juveniles. Finally, a pronounced sexual dimorphism in horn growth in Grant's gazelle likely led to confusions between the first two age classes as male horns grow faster, a young male can look like an older female when using the length of the horns as an aging criteria. This would also be the case for species with a sexual dimorphism in which only males grow horns like impala Aepyceros melampus or waterbuck Kobus defassa. Clearly, a description of the morphological changes that occur throughout the development of young herbivores (Spinage 1976 in our species, Cunningham et al. 2011, Dezeure et al. 2020 in other species) is of great value and substantially helps at reaching consistent results among different observers.

Another limitation of camera trapping in the context of reproductive studies is the level of effort required to obtain a sufficient sample size. Here, working with data from one of the world's largest and long-running camera trap studies, we ended up with a small number of sequences with juveniles $<1$ month old after appropriate data selection. We identified four main explanations for this important reduction in exploitable sequences. First and foremost, our ability to determine an individual's age class depends strongly on the photograph quality and particularly its framing and exposure. In many cases, individuals were located too far from the camera or were only partly visible, or photographs were too blurry, dark or overexposed to be scrutinized, leading to potentially significant loss of reproductive data and a high number of individuals of unknown or over-estimated age. Another potential source of information loss was species misidentification by volunteers. In our study, about $30 \%$ of the sequences labelled with Grant's gazelle were misidentified because it greatly resembles species such as impala and Thomson's gazelle which are also present in the study site. Species abundance obviously directly impacts the number of sequences collected. The abundance of the three studied species is low compared to other ungulates in the Serengeti system. Sinclair and Norton-Griffiths (1995) reported 55500 individuals topi, 20700 individuals kongoni and 6000 individuals Grant's gazelle in the 1970s, whereas the numbers of wildebeest and zebra were 720000 and 240000 individuals respectively. Finally, the anti-predator strategy of juvenile large herbivores, known as the hider-follower gradient (Lent 1974, Rutberg 1987), could influence the number of sequences containing very young individuals. While followers become active and stick with their mother just a few hours after birth, hiders stay concealed in dense vegetation during their first weeks of life. The detection probability of hiders from camera traps should then be much lower than of followers, consistent with our observation of very young topi seen in a greater proportion than kongoni and Grant's gazelle.

Volunteers' classification provides information that can reliably be used to infer the 'presence' of juveniles $<12$ months or the 'absence' of juveniles $<1$ month, as these annotations appear robust. However, because volunteers seem able to discriminate between individuals of less than one month old and the rest of the juveniles even in the absence of any stated criteria, more precise results could be achieved by asking them to differentiate between two age classes of juveniles, such as 'juvenile' and 'newborn'. The level of agreement between trained observers in the classification of age classes according to species is also a good indication of what kind of tasks could be successfully conducted by volunteers. When this agreement is low (e.g. in the identification of topi and Grant's gazelle yearlings), one could not expect volunteers to properly identify such an age class. We advise to limit the number of classes the volunteers are asked to identify, and to focus on the most recognisable ones. Another way to improve results generated via citizen science platforms could be the inclusion of detailed information, as for species identification (Swanson et al. 2015). When a volunteer detects a young on his/her photograph, he or she could be prompted with comprehensive keys to age juvenile from its morphology along with a set of reference pictures or drawings. In general, however, we would recommend to ask citizen scientists to identify newborns, i.e. individuals under one month of age versus other juveniles. To identify newborns of bovid species with horns like in our study, volunteers would have to look for the smallest individuals, with no evidence of bud horns, with specific coat color (e.g. darker coat color in Grant's gazelle in our study, or lighter coat color in wildebeest) or even with umbilical cord remnants. One difficulty is to adapt the different criteria to the every species studied.

We finally suggest evaluating volunteers' classification skills by presenting them with images of individuals of known age (captive or tagged animals for instance) and assessing their accuracy compared to labels provided by experts. Volunteers could then be assigned classification tasks adapted to their skills (e.g. species identification would belong to the easiest tasks, whereas age classification would belong to the hardest). Snapshot Safari and Zooniverse continue to create new modes of annotation that best leverage the public's interest in contributing to research, and this is a logical next step for the Snapshot Safari initiative. Overall, we find that by closely investigating the data already collected by volunteer-based programs, data collection procedures can be adjusted to enhance the contributions of citizen scientists to scientific research and conservation efforts.

\section{Data availability statement}

Pictures available from the Labelled Image Library of Alexandria - Biology and Conservation: 
<http://lila.science/datasets/snapshot-serengeti> and data from the Zenodo Repository: 10.5281/zenodo.4639695.

Acknowledgements - This work was performed using the computing facilities of the CC LBBE/PRABI. The authors thank T. Michael Anderson for maintaining the camera traps in Tanzania, Zooniverse for hosting 'Snapshot Serengeti', > 130000 online volunteers for classifying the images, the Tanzania Wildlife Research Institute and Tanzania National Parks for research permission, and the Minnesota Supercomputing Institute (<www.msi.umn.edu>) for contributing to data storage/processing and analysis.

Funding - A PhD fellowship from the university Lyon 1 attributed to LT.

Conflict of interest - The authors declare that they have no conflict of interest.

Author contributions - LT, CB and SCJ conceived the ideas and designed methodology; $\mathrm{CP}$ and $\mathrm{SH}$ organized data collection; LT, LK and MC analysed the data; LT, CB and SCJ led the writing of the manuscript. All authors contributed critically to the drafts and gave final approval for publication.

\section{References}

Agresti, A. 2002. Categorical data analysis, 3rd edn. - Wiley-Blackwell. Anderson, T. M. et al. 2016. The spatial distribution of African savannah herbivores: species associations and habitat occupancy in a landscape context. - Phil. Trans. R. Soc. B 371: 20150314.

Bartoń, K. 2019. Mumin: multi-model inference. R package ver. 1.43.10. $-<$ https://CRAN.R-project.org/package=MuMIn>.

Brosch, T. et al. 2007. That baby caught my eye... attention capture by infant faces. - Emotion 7: 685-689.

Chamaillé-Jammes, S. et al. 2014. Innate threat-sensitive foraging: black-tailed deer remain more fearful of wolf than of the less dangerous black bear even after 100 years of wolf absence. - Oecologia 174: 1151-1158.

Côté, S. D. and Festa-Bianchet, M. 2001. Birthdate, mass and survival in mountain goat kids: effects of maternal characteristics and forage quality. - Oecologia 127: 230-238.

Cunningham, P. L. et al. 2011. Morphological characteristics and ageing criteria of sand gazelle (Gazella subgutturosa marica Thomas, 1897) and their use for wildlife management. - Eur. J. Wildl. Res. 57: 865-872.

Dezeure, J. et al. 2020. Developmental transitions in body color in chacma baboon infants: implications to estimate age and developmental pace. - Am. J. Phys. Anthropol. e24118.

Fleiss, J. L. 1971. Measuring nominal scale agreement among many raters. - Psychol. Bull. 76: 378.

Golle, J. et al. 2013. Sweet puppies and cute babies: perceptual adaptation to babyfacedness transfers across species. - PLoS One 8: e58248.

Hofmeester, T. R. et al. 2019. Using by-catch data from wildlife surveys to quantify climatic parameters and the timing of phenology for plants and animals using camera traps. - Remote Sens. Ecol. Conserv. 6: 129-140.

Joshi, A. J. et al. 2009. Multi-class active learning for image classification. - In: 2009 IEEE conference on computer vision and pattern recognition. Inst. of Electronical and Electronics Engineers, pp. 2372-2379.

Karanth, K. U. et al. 2006. Assessing tiger population dynamics using photographic capture-recapture sampling. - Ecology 87 : 2925-2937.

Krishnappa, Y. S. and Turner, W. C. 2014. Software for minimalistic data management in large camera trap studies. - Ecol. Inf. 24: 11-16.

Landis, J. R. and Koch, G. G. 1977. The measurement of observer agreement for categorical data. - Biometrics 33: 159-174.
Lent, P. C. 1974. Mother-infant relationships in ungulates. - In: Geist, V. and Walther F (eds), The behaviour of ungulates and its relationship to management. IUCN Publications, Morges, Switzerland, pp. 14-55.

Luo, G. et al. 2019. Habitat use and diel activity pattern of the tibetan snowcock Tetraogallus tibetanus: a case study using camera traps for surveying high-elevation bird species. - Avian Res. 10: 4.

McNaughton, S. 1985. Ecology of a grazing ecosystem: the Serengeti. - Ecol. Monogr. 55: 259-294.

McShea, W. J. et al. 2015. Volunteer-run cameras as distributed sensors for macrosystem mammal research. - Landscape Ecol. 31: 55-66.

Meek, P. et al. 2014. Recommended guiding principles for reporting on camera trapping research. - Biodivers. Conserv. 23: 2321-2343.

Miele, V. et al. 2021. Revisiting giraffe photo-identification using deep learning and network analysis. - Methods Ecol. Evol. in press.

Norouzzadeh, M. S. et al. 2018. Automatically identifying, counting and describing wild animals in camera-trap images with deep learning. - Proc. Natl Acad. Sci. USA 115: E5716-E5725.

O'Connell, A. F. et al. 2010. Camera traps in animal ecology: methods and analyses. - Springer Science \& Business Media.

Ogutu, J. O. et al. 2008. Rainfall influences on ungulate population abundance in the Mara-Serengeti ecosystem. - J. Anim. Ecol. 77: 814-829.

Palmer, M. S. et al. 2018. Evaluating relative abundance indices for terrestrial herbivores from large-scale camera trap surveys. - Afr. J. Ecol. 56: 791-803.

Pan, W. 2001. Akaike's information criterion in generalized estimating equations. - Biometrics 57: 120-125.

Plard, F. et al. 2013. Parturition date for a given female is highly repeatable within five roe deer populations. - Biol. Lett. 9: 20120841

Quatto, P. and Ripamonti, E. 2014. raters: a modification of Fleiss' kappa in case of nominal and ordinal variables. $\mathrm{R}$ package ver. 2.0.1. $-<$ https://CRAN.R-project.org/package=raters $>$.

Riesch, H. and Potter, C. 2014. Citizen science as seen by scientists: methodological, epistemological and ethical dimensions. - Public Underst. Sci. 23: 107-120.

Rutberg, A. T. 1987. Adaptive hypotheses of birth synchrony in ruminants: an interspecific test. - Am. Nat. 130: 692-710.

Sibly, R. M. and Hone, J. 2002. Population growth rate and its determinants: an overview. - Phil. Trans. R. Soc. B 357: 1153-1170.

Sinclair, A. et al. 2000. What determines phenology and synchrony of ungulate breeding in Serengeti? - Ecology 81: 2100-2111.

Sinclair, A. R. E. and Norton-Griffiths, M. 1995. Serengeti: dynamics of an ecosystem. - Univ. of Chicago Press.

Spinage, C. 1976. Age determination of the female Grant's gazelle. - Afr. J. Ecol. 14: 121-134.

Swanson, A. et al. 2015. Snapshot Serengeti, high-frequency annotated camera trap images of 40 mammalian species in an African savanna. - Sci. Data 2: 150026.

Swanson, A. et al. 2016. A generalized approach for producing, quantifying and validating citizen science data from wildlife images. - Conserv. Biol. 30: 520-531.

Vanbelle, S. and Albert, A. 2008. A bootstrap method for comparing correlated kappa coefficients. - J. Stat. Comput. Simul. 78: 1009-1015.

Visser, M. E. and Both, C. 2005. Shifts in phenology due to global climate change: the need for a yardstick. - Proc. R. Soc. B 272: 2561-2569.

Wearn, O. and Glover-Kapfer, P. 2017. Camera-trapping for conservation: a guide to best-practices. - WWF Conserv. Technol. Series 1: 2019-04.

Wilson, D. E. and Mittermeier, R. A. 2011. Handbook of the mammals of the world. Vol. 2. Hoofed mammals. - Lynx Edicions.

$\mathrm{Xu}, \mathrm{C}$. et al. 2018. wgeesel: weighted generalized estimating equations and model selection. $\mathrm{R}$ package ver. 1.5. - < https:// CRAN.R-project.org/package $=$ wgeesel $>$. 